\title{
Study on the Tibetan Language Learning of Tibetan and Chinese Bilingual Students in Physics
}

\author{
Bingbing Chen \\ Sichuan Minzu College, Kangding, Sichuan, 626001
}

Keywords: Tibetan-Chinese bilingual students; Tibetan; learning

\begin{abstract}
With literature review, interviews, and questionnaire survey methods, this paper explores and studies Tibetan-Chinese bilingual students in physics in Tibetan language learning. This paper firstly summarizes the status of bilingual education in Ganzi Tibetan Autonomous Region and the status of bilingual teachers. Then it analyzes the status quo of bilingual students' Tibetan language learning ability according to a questionnaire survey, finds out the reasons, and finally formulates corresponding solutions in light of the actual situation of the school. Through the exploration and research, it is expected to find an effective method for Tibetan bilingual study of bilingual students and provide some experience and reference for the study of similar issues in the future.
\end{abstract}

\section{Introduction}

Due to many factors, such as history and society, the Ganzi Tibetan area has become old, young, poor and poor areas. The economic backwardness directly leads to the lag in culture, education and social development. The traditional production methods in some areas have not yet been fundamentally changed. In the vast agricultural and pastoral areas of the Ganzi Tibetan Autonomous Region, the characteristics of the Tibetan people's native language communication are very prominent. The use of Chinese language in these regions is relatively low, and bilingual education in Tibetan and Chinese is imperative.

According to statistics, by the end of 2016, there were 968 schools at various levels in the Ganzi Tibetan Region, including 356 kindergartens, 555 primary schools, 52 secondary schools, 1 special education school, 3 secondary professional schools, and 1 higher education institution. The. There are 12,243 full-time teachers throughout the state, including 1,100 kindergarten teachers, 7,118 primary school teachers, 3,170 ordinary high schools, 131 vocational middle schools, 20 special educations, and 289 secondary specialized schools. However, in this huge team, there are less than $10 \%$ of bilingual teachers who can truly teach in Tibetan and Chinese, and bilingual teachers of science majors are in serious shortage. At present, the failure to introduce bilingual teachers and the introduction of them have severely constrained the development of education in the Ganzi Tibetan Region, seriously restricting the progress and quality of full coverage of the 15-year free compulsory education in the Ganzi Tibetan Region. According to the characteristics of bilingual teachers in Sichuan Zang district requiring "multiple varieties and small batches", in 2016, the Ganzi Prefecture Education Bureau asked the School of Physical Education of recruiting ethnic minority students to request the training of qualified personnel, requesting that our school be an annual The orientation training of about 30 people physics professional bilingual teacher.

\section{Analysis of the Status Quo of Tibetan Language in Students}

In September 2016, our university formally enrolled the first physics-based Tibetan-Chinese bilingual students in a total of 20 students, including 12 boys and 8 girls, 16 students from Ganzi, 2 from Aba, and 2 from Liangshan. They are all Tibetan and Canadian candidates, which means that in addition to language, mathematics, English, and comprehensive scores, students will also be asked to add one Tibetan section. After the students entered the university, we conducted a questionnaire survey on their family environment, language environment, and Tibetan and Tibetan language learning in the primary and secondary schools based on the research needs of the 
school-level education reform project. We now summarize the survey into three major issues. The analysis of the survey data hopes to have a certain understanding of the Tibetan language level of the students. At the same time, they hope that the data analysis can be used to find the reasons for the poor Tibetan language level and provide the basis for the formulation of countermeasures.

Table 1 Results Analysis Table

\begin{tabular}{|c|c|c|c|c|c|c|c|c|c|c|}
\hline Score & $0-9$ & $10-19$ & $20-29$ & $30-39$ & $40-49$ & $50-59$ & $60-69$ & $70-79$ & $80-89$ & $90-100$ \\
\hline Num & 5 & 9 & 1 & 2 & 0 & 1 & 1 & 1 & 0 & 0 \\
\hline Percent & $25 \%$ & $45 \%$ & $5 \%$ & $10 \%$ & $0 \%$ & $5 \%$ & $5 \%$ & $5 \%$ & $0 \%$ & $0 \%$ \\
\hline
\end{tabular}

According to the scores of Tibetan in the college entrance examination, the average score of the class (out of 100 points) is 21.75 , the highest score is 72 , and the lowest score is 8 points. From the above table, we can see that the student's Tibetan level is relatively poor, the pass rate is only $10 \%$, and from the point of view of the score distribution, less than 20 points account for $70 \%$, which also includes guessing when doing multiple choice questions. And the situation of scoring.

Table 2 Language Environment Survey

\begin{tabular}{|c|c|c|c|c|}
\hline No. & Issue & Option & Result & Percent \\
\hline \multirow{2}{*}{1} & \multirow{2}{*}{$\begin{array}{c}\text { Whether your hometown } \\
\text { language is Chinese, local or } \\
\text { Tibetan }\end{array}$} & $\begin{array}{c}\text { A. locale } \\
\text { language }\end{array}$ & 5 & 25 \\
\cline { 3 - 5 } & $\begin{array}{c}\text { B. Tibetan } \\
\text { language }\end{array}$ & 3 & 15 \\
\cline { 3 - 5 } & & C. Chinese & 12 & 60 \\
\hline \multirow{2}{*}{2} & $\begin{array}{c}\text { Do you speak Tibetan in your } \\
\text { family members' lives? }\end{array}$ & A.Y & 6 & 30 \\
\cline { 3 - 5 } 3 & $\begin{array}{c}\text { Do you like to speak Tibetan in } \\
\text { your daily study and life? }\end{array}$ & B.N & 14 & 70 \\
\cline { 3 - 5 } & & A.Y & 7 & 35 \\
\hline
\end{tabular}

In the survey of the language environment, $60 \%$ of the students' native language is Chinese, Tibetan only 15\%, local languages (local dialects, not Tibetan) 25\%; family members speak 30\% Tibetan, Chinese 70\% In the usual Tibetan language survey, 35\% of the respondents chose to like it. It can be seen that they still showed a certain interest in Tibetan. From the analysis, it can be found that most students have less opportunities to listen and speak Tibetan in their lives. This means that the Tibetan language environment is not strong, and even some places never speak Tibetan.

From the above table, it was found that only $25 \%$ of the students had studied Tibetan before university and 75\% had entered the university before they started systematic learning. In the Tibetan study time survey, $55 \%$ of the students' study time was 0 years. In the $30 \%$ of the following years, only $15 \%$ of students have studied Tibetan for more than six years. The time for students to study Tibetan before university is generally short, which may also be the main reason for the poor performance of Tibetan students.

The above table investigates students' Tibetan listening, speaking, reading and writing abilities. We find that $80 \%$ of the students' listening ability is not in the listening level, they are $5 \%$ in the general and $1 \%$ in the listening, and only 10 in the better. \%; Tibetan speaking, $65 \%$ wouldn't, $15 \%$ and $15 \%$ would be better, and 5\% would feel sensible. In reading, $65 \%$ wouldn't read it. $15 \%$ of the students have a good $10 \%$ reading ability and a good feeling. In Tibetan writing, $80 \%$ will not, and $10 \%$ will feel a bit different. From the four aspects of analysis, students' listening, speaking, reading and writing skills are still low, and 65\%-80\% of students are completely absent in these four aspects, which means that the Tibetan language is based on The ability is zero. 
Table 3 Use of Tibetan to Conduct Other Subjects Teaching Survey

\begin{tabular}{|c|c|c|c|c|}
\hline No. & Issue & Section & Result & Percent \\
\hline \multirow{3}{*}{1} & \multirow{3}{*}{$\begin{array}{l}\text { In your learning experience } \\
\text { (excluding universities), have } \\
\text { you used any other subjects } \\
\text { taught in Tibetan, such as } \\
\text { mathematics, physics, and } \\
\text { politics? }\end{array}$} & A.N.A & 19 & 95 \\
\hline & & B. Part of & 1 & 5 \\
\hline & & C.All & 0 & 0 \\
\hline
\end{tabular}

\section{Formulate Corresponding Personnel Training Programs and Increase Tibetan School Hours}

This is the curriculum arrangement of the Tibetan language in the Talent Development Program for the 2016 Physical Education (Bilingual-Chinese Bilingual) program, which includes the semester for the course and the corresponding amount of time for the course. Among them, the "Basic Tibetan" course mainly introduces Tibetan knowledge in a systematic and progressive manner from the Tibetan foundation. The "Tibetan Information Processing" course requires students to understand the basic concepts and basic principles of the Tibetan information processing technology, focusing on the commonly used Tibetan software systems. Use methods and operating skills to develop students' basic literacy in Tibetan information processing. The "Tibetan Orthography" course introduces the regular use, writing, and related grammar of Tibetan. The "Selected Works of Tibetan Languages" course mainly introduces Tibetan culture and achievements in the development process, and deepens students' understanding of the cultural background. The "Physics Term Tibetan-Chinese Translation" course mainly combines junior middle school physics textbooks (Tibetan version) and contrasts physical terminology. Translation and interpretation. From the point of view of the opened and ongoing teaching of courses, teachers can complete teaching tasks according to the teaching plan and outline of the course, mainly adopting classroom teaching and arranging corresponding exercises after class to consolidate the learning effect. Students choose their own time after class to review the course. . However, from the perspective of students' learning effects, the situation is not satisfactory. The progress of Tibetan students with poor basics is not obvious. The reason is that some students do not have enough concentration in class, and they basically do not have time to consolidate after class. There are also courses. The amount of class time is less. Therefore, in the future development of the talent training program, it is possible to increase the hours of the Tibetan language courses and ensure that there are 2 lessons per day on the basis of the Tibetan language. The teachers pay attention to the degree of concentration of student learning in the class and strengthen the monitoring of students' learning effects in the ordinary teaching. Students are required to use Tibetan language for a certain period of time after class.

Pursuant to the "Ganzi State Order-Oriented Talent Cultivation Cooperation Agreement," in order to further improve the ability of bilingual students to listen, speak, read and write in the Tibetan language, after the proposal for admission, the Tibetan teachers are specifically invited to use the holiday time to focus on Tibetan students. In the training, 28 lessons in "Basic Tibetan Studies" and 16 lessons in "Tibetan Writings Act" focused on strengthening the learning and training of students' Tibetan knowledge and achieved certain results. However, because of the short time, the effect achieved was limited. It is recommended to increase the training time in the future holiday training center. At the same time, it should also pay attention to the detection of teaching effects to understand the students' knowledge of knowledge.

Through the analysis of the questionnaire, we have found that the polarization of students' Tibetan language is quite serious. Looking at the effects of classroom training and holiday-focused training, the progress of well-based students is great, and the progress of poor foundation is slow. Students in the classroom showed two different reactions and states. Based on the above issues, we conducted a group focused counseling study. In order to make our tutoring targeted and able to meet 
the needs of students at different levels, we divided the students in the class into three levels according to the level of Tibetan language for group counseling. Four well-educated classmates were selected as the small teacher (leader) of the tutoring study. Each young teacher developed corresponding learning tasks and plans based on the students' specific circumstances at the beginning of each semester, and conducted two sessions each week. Counselling, one and a half hours at a time.

This level is the lowest level. After they enter the university for one year of "basic Tibetan language" courses, the Tibetan level can basically reach the level of the second grade of elementary school. Although the spelling will not have much problems, It is still very difficult for them to make sentences in Tibetan. This is mainly because there is a big difference between Tibetan and Chinese. These students use Chinese to communicate with people around them at home. Therefore, they have been thinking and expressing in Chinese logical thinking since childhood, so it is necessary to teach them some of the Tibetan language expressions that are commonly used in daily life. Grasping the Tibetan way of thinking. Tibetan is as rigorous as English, and it is difficult to master. Its learning is often synchronised with the memory of words and the learning of grammar. We also follow this rule to organize counseling.

This level is in the middle level. Although they are a little bit better than the Tibetan level in the first level, the actual difference is not large. The only difference is that they speak Tibetan but they do not understand Tibetan. So we Instead of teaching them Tibetan logical thinking, they can only teach grammar and words.

This level of students has a good level of Tibetan language. Most of the students have studied Tibetan in primary or secondary schools. These students mainly study grammar. The rest of the time requires them to read more Tibetan books. Questions can be directed to the teacher or a good Tibetan student.

There is a direct connection between the continuity of Tibetan language learning and the development of interest. Therefore, in order to better stimulate students' enthusiasm and interest in learning, we hold examinations or Tibetan knowledge contests at regular intervals in the class, and press the results of the examination to score Outstanding classmates give rewards. Of course, students who perform poorly or do not achieve the desired results will be punished accordingly. Through one-year group tutoring, most of the students have made some progress while the team leader is seriously responsible and the students' own efforts. However, some problems still appear during the process of counseling and learning. Some of the students are still not important for learning Tibetan. Sex pays attention, and she is absent from teaching, not serious, and does not want to spend time learning after class. It is also necessary to think more about the pertinence of knowledge in teaching. In response to the problems we have encountered, we will adjust our teaching plans in a timely manner and strengthen the students' guidance in learning Tibetan. We will make them realize that learning Tibetan is closely related to their own work.

\section{Conclusion}

The Tibetan-Chinese bilingual students enrolled in the school's physical education are a very important measure for strengthening the implementation of bilingual teachers in the Ganzi Tibetan Region. However, due to the poor foundation of students' Tibetan language and the constraints of the actual conditions of the school, we are facing challenges in the cultivation process. There are many difficulties and no ready-made experience for our reference. We only face the reality and do our utmost efforts. Teachers think more about teaching methods, teaching content and the formulation of talent training programs. Students start from their own time and spend more time to strengthen the basic knowledge of Tibetan language learning. Competent bilingual teaching tasks, to contribute to the education of the Ganzi Tibetan region.

\section{Acknowledgements}

Fund Project: Sichuan Minzu Collge Education Reform Project “Tibetan-Chinese Bilingual 
Major Student Tibetan Learning Exploration Research”, Project No. 2017xjjg10.

\section{References}

[1] Li Nengwu. Problems of Tibetan-Chinese bilingual teaching in Sichuan Institute for Nationalities and its countermeasures [J]. Journal of Sichuan University for Nationalities, 2015. (2):94-97

[2] Ye Xiaobin. Educational Status and Thinking of Ganzi Tibetan Area[J]. Journal of Southwest Jiaotong University (Social Science Edition), 2015. (8):94-99

[3] Liu Mengzhen. Problems and Countermeasures of Bilingual Teaching in Ganzi Tibetan Autonomous Prefecture [J]. Education and Teaching Forum, 2015. (5): 259-260

[4] Li Shicun et al. Suggestions on Improving the Teaching Quality of Normal University Students of Science in Tibet [J]. Western Quality Education, 2017. (2): 13-14

[5] Yan Muchu. Tibetan-Chinese Bilingual Drive, Liberal Arts, Science and Technology: An Exploration of the Road to Bilingual-Chinese Bilingual Education[J]. Journal of Aba Teachers College, 2015. (4):121-125 